\title{
Measurement of picosecond lifetimes in neutron-rich Xe isotopes
}

S. Ilieva, ${ }^{1, *}$ Th. Kröll,,${ }^{1}$ J.-M. Régis, ${ }^{2}$ N. Saed-Samii, ${ }^{2}$ A. Blanc,${ }^{3}$ A. M. Bruce, ${ }^{4}$ L. M. Fraile,${ }^{5}$ G. de France, ${ }^{6}$ A.-L. Hartig, ${ }^{1}$ C. Henrich, ${ }^{1}$ A. Ignatov, ${ }^{1}$ M. Jentschel, ${ }^{3}$ J. Jolie, ${ }^{2}$ W. Korten, ${ }^{7}$ U. Köster,${ }^{3}$ S. Lalkovski,,${ }^{4,8}$ R. Lozeva, ${ }^{9}$ H. Mach,${ }^{5, \dagger}$ N. Mărginean, ${ }^{10}$ P. Mutti, ${ }^{3}$ V. Paziy, ${ }^{5}$ P. H. Regan, ${ }^{11,12}$ G. S. Simpson, ${ }^{13,14}$ T. Soldner, ${ }^{3}$ M. Thürauf, ${ }^{1}$ C. A. Ur, ${ }^{10}$ W. Urban, ${ }^{15}$ and N. Warr ${ }^{2}$

${ }^{1}$ Institut für Kernphysik, Technische Universität Darmstadt, Germany

${ }^{2}$ Institut für Kernphysik, Universität zu Köln, Germany

${ }^{3}$ Institut Laue-Langevin, Grenoble, France

${ }^{4}$ School of Computing, Engineering and Mathematics, University of Brighton, United Kingdom

${ }^{5}$ Universidad Complutense, CEI Moncloa, Madrid, Spain

${ }^{6}$ Grand Accélérateur National d'Ions Lourds, Caen, France

${ }^{7}$ CEA Saclay, France

${ }^{8}$ Faculty of Physics, University of Sofia, Bulgaria

${ }^{9}$ Institut Pluridisciplinaire Hubert Curien and the University of Strasbourg, Strasbourg, France

${ }^{10}$ Horia Hulubei National Institute of Physics and Nuclear Engineering, Bucharest, Romania

${ }^{11}$ Department of Physics, University of Surrey, Guildford, United Kingdom

${ }^{12}$ National Physical Laboratory, Teddington, Middlesex, TW11 OLW, United Kingdom

${ }^{13}$ University of the West of Scotland, Paisley, United Kingdom

${ }^{14}$ Laboratoire de physique subatomique et de cosmologie de Grenoble (LPSC), Grenoble, France

${ }^{15}$ Faculty of Physics, University of Warsaw, Warsaw, Poland

(Received 25 July 2016; published 1 September 2016)

\begin{abstract}
Background: Lifetimes of nuclear excited states in fission fragments have been studied in the past following isotope separation, thus giving access mainly to the fragments' daughters and only to long-lived isomeric states in the primary fragments. For the first time now, short-lived excited states in the primary fragments, produced in neutron-induced prompt fission of ${ }^{235} \mathrm{U}$ and ${ }^{241} \mathrm{Pu}$, were studied within the EXILL\&FATIMA campaign at the intense neutron-beam facility of the Institute Laue-Langevin in Grenoble.

Purpose: We aim to investigate the quadrupole collective properties of neutron-rich even-even ${ }^{138,140,142} \mathrm{Xe}$ isotopes lying between the double shell closure $N=82$ and $Z=50$ and a deformed region with octupole collectivity.

Method: The $\gamma$ rays emitted from the excited fragments were detected with a mixed array consisting of 8 HPGe EXOGAM Clover detectors (EXILL) and $16 \mathrm{LaBr}_{3}(\mathrm{Ce})$ fast scintillators (FATIMA). The detector system has the unique ability to select the interesting fragment making use of the high resolution of the HPGe detectors and determine subnanosecond lifetimes using the fast scintillators. For the analysis the generalized centroid difference method was used.

Results: We show that quadrupole collectivity increases smoothly with increasing neutron number above the closed $N=82$ neutron shell. Our measurements are complemented by state-of-the-art theory calculations based on shell-model descriptions.

Conclusions: The observed smooth increase in quadrupole collectivity is similar to the evolution seen in the measured masses of the xenon isotopic chain and is well reproduced by theory. This behavior is in contrast to higher $Z$ even-even nuclei where abrupt change in deformation occurs around $N=90$.
\end{abstract}

DOI: 10.1103/PhysRevC.94.034302

\section{INTRODUCTION}

Many of the low-lying structures of even-even nuclei can be described by collective degrees of freedom. The region of neutron-rich nuclei above the closed shells $Z=50$ and $N=82\left({ }^{132} \mathrm{Sn}\right)$ is of particular interest as both quadrupole (reflection symmetric mode) and octupole (reflection asymmetric mode) collective properties can be studied in the structure of these nuclei [1-3]. The electromagnetic properties of the

\footnotetext{
*silieva@ikp.tu-darmstadt.de

${ }^{\dagger}$ Deceased.
}

nuclei, like $B(E 2)$ transition strengths (linked to the wave functions of the initial and final states) and $g$ factors (related to the microscopic structure of a single state), are good probes for the degree of collectivity of nuclear excitations.

Single-particle and mean-field theoretical approaches can be applied for nuclei in this mass region. For nuclei close to the

valley of stability, the $T=0$ component of the protonneutron interaction results in similar deformation for protons and neutrons and leads to an isoscalar $2_{1}^{+}$state [4]. Adding more valence neutrons to these nuclei, the additional neutrons might decouple from the core, resulting in a different deformation. The lowest spin-parity $2^{+}$state would have an isovector admixture which lowers its energy while the $B\left(E 2 ; 0_{\mathrm{gs}}^{+} \rightarrow 2_{1}^{+}\right)$ 
value which is dominated by the proton core remains nearly the same. Studying the evolution of the $B\left(E 2 ; 0_{\mathrm{gs}}^{+} \rightarrow 2_{1}^{+}\right)$values with increasing neutron number gives insight on the onset of quadrupole collectivity and deformation.

Octupole deformation in the ground state of atomic nuclei is expected to occur at proton and neutron numbers close to 56,88 , and 136 [5,6]. Manifestations of strong octupole correlations were observed in the regions of heavy $\mathrm{Xe}$ and Ba nuclei $(Z \approx 56$ and $N \approx 88)$ [2,7]. Evidence for octupole correlations also appears in the context of mass measurements. The two-neutron separation energies, $S_{2 n}$, for neutron-rich xenon isotopes decrease rather smoothly with increasing neutron number. That is in contrast to isotopic chains of heavier elements where deformation sets in around $N=90$ and $S_{2 n}$ values flatten. The inclusion of an octupole degree of freedom in this region considerably improves the agreement between measured and calculated masses [8].

Direct electro-magnetic transition rate and related excited state lifetime measurements are an excellent tool to study the collective nuclear properties. In this paper we focus on the quadrupole collective properties of the neutron-rich even-even xenon isotopes. The $B\left(E 2 ; 0^{+} \rightarrow 2^{+}\right) \uparrow$ transition strength for the excitation of the first $2^{+}$state was previously determined only in the ${ }^{140} \mathrm{Xe}$ isotope. The existing values $B(E 2 \uparrow)=$ $0.538(17) e^{2} b^{2}$ [2] and $B(E 2 \uparrow)=0.335(7) e^{2} b^{2}$ [9] contradict each other and the latter cannot be reproduced by theory. Our study resolves this puzzle and the lifetimes determined for other positive-parity states will also be presented and discussed.

\section{THE EXILL\&FATIMA EXPERIMENT}

The structure of fission fragments has been a subject of many previous investigations (e.g., Ref. [10]). In most cases, the decay products of the fragments were studied following an isotope separation. The EXILL\&FATIMA experiment is the first prompt fission-fragment $\gamma$-ray spectroscopy experiment using the fast-timing technique. In former experiments, higherlying states in the fragments were studied using direct timing with Ge detectors [11]. Due to the limited-timing performance of Ge detectors, only lifetimes longer than several ns can be measured. For shorter lifetimes in the ps to ns region, the Recoil distance Doppler-shift method (plunger) was used where $\gamma$ rays emitted in flight and at rest are detected and the lifetime can be determined using the Doppler shift of the $\gamma$ rays [12]. The $\gamma$ rays emitted in flight are Doppler broadened, which results in broader peaks. Thus, this technique is limited to studies of fission fragments with high production rate. Spectroscopy of excited states and direct-timing measurements were applied on the $\beta$-decay daughters of the fission fragments, in which case mostly low-spin states were studied. In most cases fast $\mathrm{BaF}_{2}$ detectors were used (e.g., Ref. [2]) which have inferior energy resolution as compared to the $\mathrm{LaBr}_{3}(\mathrm{Ce})$ detectors used in our study, and therefore simple spectra with only few transitions are required.

The EXILL\&FATIMA experiment was performed at the Institute Laue-Langevin (ILL) in Grenoble, which provides the world highest flux of thermal- or cold-neutron beams from its experimental reactor. The fission target was placed in the

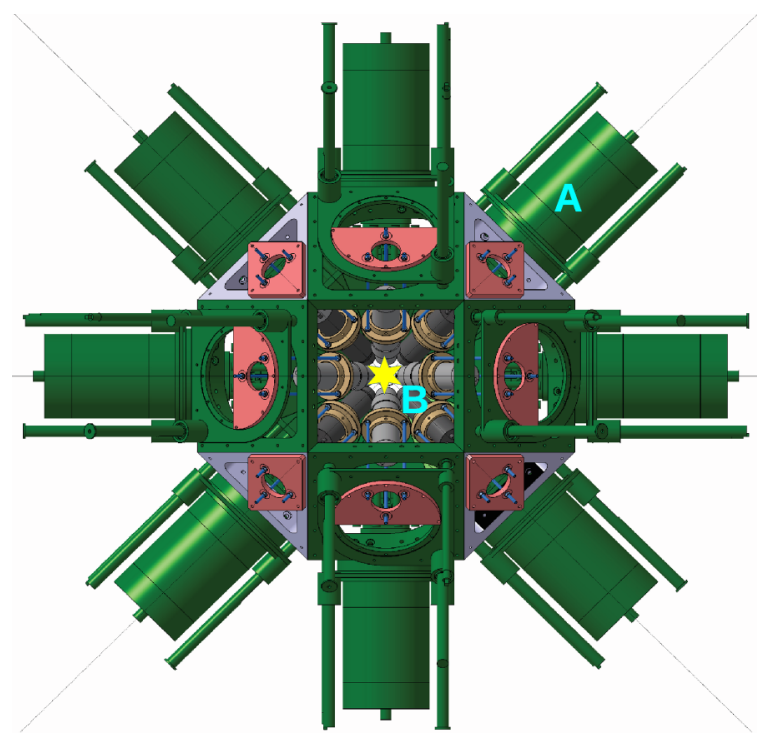

FIG. 1. CAD design of the EXILL\&FATIMA spectrometer equipped with (A) bismuth germanate Compton-suppressed EXOGAM Ge-clover detectors and (B) $\mathrm{LaBr}_{3}(\mathrm{Ce})$-scintillator detectors. The target position is indicated with a star in the center of the array. The neutron beam is in direction perpendicular to the plane of the figure.

center of the EXILL\&FATIMA detector array (Fig. 1). Two different targets were used, $0.674 \mathrm{mg}{ }^{235} \mathrm{U}$ and $0.3 \mathrm{mg}{ }^{241} \mathrm{Pu}$, each of them sandwiched between two $24-\mu \mathrm{m}$-thick layers of beryllium. The target surface was about $1 \mathrm{~cm}^{2}$. The fission was induced by a cold-neutron beam with a flux $\Phi \approx 10^{8} \mathrm{n} /\left(\mathrm{cm}^{2} \mathrm{~s}\right)$ from the collimated neutron guide PF1B [13]. The fission of these actinides gives access to exotic nuclei in the mass region $80 \leqslant A \leqslant 160$.

The $\gamma$ rays were detected with the EXILL\&FATIMA array [14], which is a unique mixed array of $\mathrm{HPGe}$ and $\mathrm{LaBr}_{3}(\mathrm{Ce})$ detectors. The EXOGAM at ILL (EXILL) array comprised eight Clover detectors with four HPGe crystals each. They were placed in a ring at $90^{\circ}$ with respect to the beam line. The FATIMA detector array contained $16 \mathrm{LaBr}_{3}(\mathrm{Ce})$ fast scintillators, placed in two groups of eight detectors in forward and backward directions, respectively. The $\mathrm{LaBr}_{3}(\mathrm{Ce})$ crystals were cylindrical with a diameter of 1.5 inches; 8 crystals had length 1.5 inches and the other 8 crystals were 2 inches long. The efficiencies of the EXILL and FATIMA arrays were $\approx 5 \%$ and $\approx 4 \%$ at $662 \mathrm{keV}$, respectively [14].

During the experiment triggerless time-stamped data were recorded. The time stamping was done using synchronized CAEN V1724 100-MHz digitizers [14]. Data were taken with a rate of $\sim 10-15 \mathrm{kHz}$ per detector, which resulted in a very large amount of data from the experiment $(\approx 12 \mathrm{~TB})$. To limit the size of the data files, the data were stored in separate files, each one corresponding to a measurement time of $5 \mathrm{~min}$.

The first part of the calibration procedure was a detector stability check. In the energy range of interest (up to 2 $\mathrm{MeV})$, the $\mathrm{Ge}$ detectors were stable while the $\mathrm{LaBr}_{3}(\mathrm{Ce})$ detectors showed an amplitude drift with time (see Fig. 2). This drift was caused by the high rate which heated the 


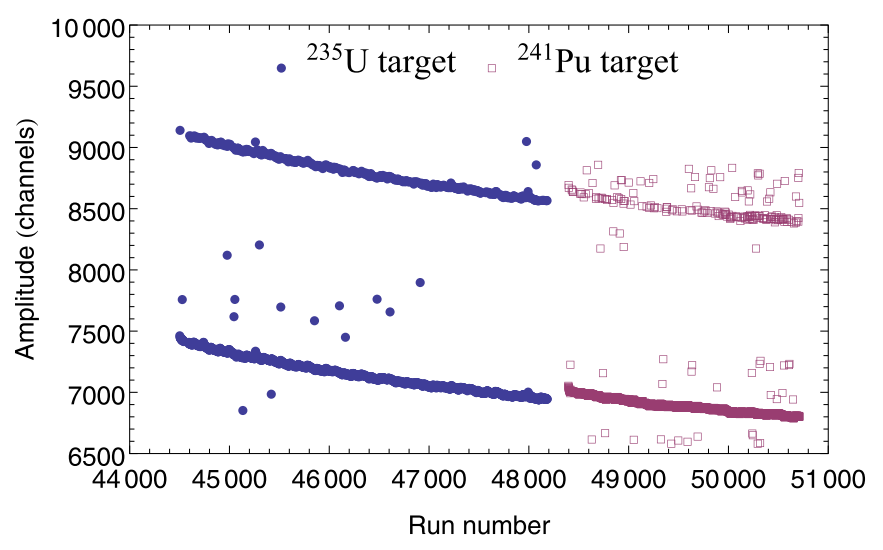

FIG. 2. Example of the drift of the amplitude of the $\operatorname{LaBr}_{3}(\mathrm{Ce})$ signals with time in one of the detectors from the measurements with two different target materials. The time scale corresponds approximately to 24 days of beam time. The energies of the transitions in this example correspond to $\sim 1.78 \mathrm{MeV}$ and $\sim 2.23 \mathrm{MeV}$.

photomultiplier tubes used to read out the scintillators leading to a decrease in the signal amplitude. To correct for the amplitude drift, the positions of several peaks in the spectra obtained for each detector and each file were determined using an automatic search algorithm. This algorithm failed for files with low statistics (scattered points in Fig. 2); in this case the peak positions had to be determined manually. Afterwards, a reference spectrum was chosen and the positions of the lines in the rest of the data files were aligned to the positions of the lines in the reference spectrum. Recalibration functions were determined for each detector and file and the amplitude gains of the detectors were matched file by file. For the calibration of the data with a ${ }^{235} \mathrm{U}$ target a second-order polynomial and for the data with $\mathrm{a}^{241} \mathrm{Pu}$ target a linear function was used.

The energy calibration of the Ge detectors was done using a standard ${ }^{152} \mathrm{Eu}$ source. Due to the amplitude drift of the $\mathrm{LaBr}_{3}(\mathrm{Ce})$ signals, this measurement could not be used for their energy calibration. For this purpose, strong lines in the fission spectrum, after gain matching, were chosen and then calibrated using the energies measured in the Ge detectors for the same lines. The relative energy resolution of the FATIMA array was $3.3 \%$ at $662 \mathrm{keV}$ [14].

As a next step the data were sorted in events offline. For the event building a 120-ns time window was used. The data were analyzed with the socov2 software developed at the Institute of Nuclear Physics (IKP) in Cologne, Germany [15]. During the event building, events where pairs of neighboring $\mathrm{LaBr}_{3}(\mathrm{Ce})$ crystals fired were excluded as there were no means of distinguishing real double hits from $\gamma$ rays Compton scattered between neighboring crystals. Prompt $\gamma$-ray cascades in the nuclei of interest were selected from these events using triple $\mathrm{Ge}-\mathrm{LaBr}_{3}(\mathrm{Ce})-\mathrm{LaBr}_{3}(\mathrm{Ce})$ or quadruple $\mathrm{Ge}-\mathrm{Ge}-$ $\mathrm{LaBr}_{3}(\mathrm{Ce})-\mathrm{LaBr}_{3}(\mathrm{Ce})$ conditions. The energy resolution of the $\mathrm{Ge}$ detectors allowed us to set precise energy gates in order to select the cascade of interest in a particular nucleus. This coincidence condition was necessary due to the large amount of populated fission fragments. Alternatively, the $\mathrm{Ge}$ selection gate could be placed on the ground-state transition of one of the complementary fission partners. This is not of a big advantage as in this case only part of the statistics for the nucleus of interest is selected although sometimes cleaner spectra are obtained. The time difference between the $\gamma$ rays feeding and decaying from a state could be directly measured in a ps-to-ns range thanks to the time performance of the $\operatorname{LaBr}_{3}(\mathrm{Ce})$ scintillators. The measured full width at half maximum (FWHM) of the timing response of the different couples of scintillators was $210-300$ ps for the ${ }^{60} \mathrm{Co}$ lines, the differences mainly coming from the different sizes of the crystals and also due to small variations in the Ce doping [14].

\section{DETERMINATION OF THE LIFETIMES-THE GENERALIZED CENTROID DIFFERENCE METHOD}

The lifetimes of the excited states were determined using the generalized centroid difference method (see J.-M. Régis et al. [16] and references therein). This method provides two independent delayed and antidelayed time spectra obtained as a superposition of $N(N-1) / 2$ calibrated $\gamma-\gamma$ time-difference spectra of a fast-timing array consisting of $N$ detectors. The two time spectra correspond to forward and reverse gating on a specific $\gamma-\gamma$ cascade and the centroid difference of the two time spectra provides a picosecond-sensitive and mirrorsymmetric observable of the whole fast-timing array.

The centroid of a time distribution generated as the time difference between consecutive $\gamma$ rays, measured using a start and stop $\gamma$-ray detector, can be used to determine the lifetime of the state connecting the two $\gamma$ rays. A delayed time distribution $D(t)$ is obtained when the feeding $\gamma$ ray is detected by the start detector and the decay by the stop detector. When no background is present, $D(t)$ is a convolution of the normalized prompt response function of the setup $P(t)$ with an exponential decay:

$$
D(t)=n \lambda \int_{-\infty}^{t} P\left(t^{\prime}-t_{0}\right) e^{-\lambda\left(t-t^{\prime}\right)} d t^{\prime}, \quad \lambda=1 / \tau
$$

where $n$ is the total number of detected $\gamma-\gamma$ events, $\lambda$ is the transition probability and $\tau$ is the mean lifetime of the nuclear state [16]. For lifetimes shorter than the FWHM of the prompt response function, the lifetime of the state can no longer be determined from the slope of the exponential decay, so instead the centroid shift method [17] is used:

$$
\tau=C^{D}-C_{\text {stop }}^{P}\left(E_{\gamma}\right),
$$

where $C^{D}$ is the centroid of the delayed time distribution and $C_{\text {stop }}^{P}\left(E_{\gamma}\right)$ represents the energy-dependent zero time (centroid of the PRF) related to the stop detector. The centroid or "center of gravity" is given by:

$$
C^{D}=\frac{\int t D(t) d t}{\int D(t) d t} \quad \text { and } \quad C^{P}=\frac{\int t P(t) d t}{\int P(t) d t} .
$$

When the functions of the two detectors are interchanged (the start is given by the decay $\gamma$ ray and, the stop by the $\gamma$ ray feeding the state of interest) an antidelayed spectrum is obtained and the lifetime of the state is given by:

$$
\tau=C_{\text {start }}^{P}\left(E_{\gamma}\right)-C^{\mathrm{AD}} .
$$


The critical point for such a measurement is the determination of the prompt centroid as it is dependent on the timing response of the setup, i.e., the time-walk characteristics $T\left(E_{\gamma}\right)$. Within the mirror-symmetric centroid difference method [18] it was shown that the centroid difference of a two-detector system is mirror symmetric with respect to the energy difference of the two $\gamma$ rays from the cascade. This mirror symmetry provides additional data points for the precise calibration of the "prompt response difference" (PRD), which represents the linearly combined $\gamma-\gamma$ time-walk characteristics of the two detector timing system. This procedure eliminates the necessity to calibrate the timing responses of each detector separately and reduces possible systematic errors. Thus, the lifetime, or the PRD, respectively, can be determined from the relation:

$$
\begin{gathered}
\Delta C=\mathrm{PRD}+2 \tau \text { with } \\
\Delta C=C^{D}-C^{\mathrm{AD}} \text { and } \\
\mathrm{PRD}=C_{\text {stop }}^{P}\left(E_{\gamma}\right)-C_{\text {start }}^{P}\left(E_{\gamma}\right) .
\end{gathered}
$$

For a detection system of $N$ detectors, the time difference between the delayed (stop) and antidelayed (start) events is statistically distributed around the mean $\overline{\Delta C}$ and $\overline{\text { PRD }}$ independent of the detector-detector combination. This represents the essence of the generalized centroid difference method [16].

For the calibration of the energy-dependent zero-time response of the detector array (PRD curve), prompt transitions $(\tau<1 \mathrm{ps})$ and transition from states with precisely known lifetimes $(\tau<100 \mathrm{ps})$ from the decay of ${ }^{152} \mathrm{Eu}$ and from the neutron-capture reaction ${ }^{48} \mathrm{Ti}\left(n_{\mathrm{th}}, \gamma\right)^{49} \mathrm{Ti}$ were used. A detailed description of the PRD calibration procedure is given in Ref. [19]. The derived prompt response differences were fitted with the function:

$$
\operatorname{PRD}\left(E_{\gamma}\right)=\frac{a}{\sqrt{E_{\gamma}+b}}+c E_{\gamma}+d E_{\gamma}^{2}+e
$$

with fit parameters $a, b, c, d$, and $e$, and the result is shown in Fig. 3 (top). The achieved overall PRD uncertainty within $3 \sigma$, taking into account statistical uncertainties, in the energy region $40 \mathrm{keV}$ to $1800 \mathrm{keV}$ is $5 \mathrm{ps}$ as seen from the PRD fit residual [Fig. 3 (bottom)].

In an experiment with a fission target many fragments are produced emitting many different $\gamma$ rays which create a lot of background. In such a case the experimental conditions are not clean and it is very important to account for the contribution of the background to the centroid difference. In a fission fragment measurement, the background mainly consists of Compton background generated from primary $\gamma$ rays which may belong to different nuclei with different time responses and are overlayed with the time response of the state of interest. Below about $400 \mathrm{keV}$, additional largely delayed background is obtained by the detection of low-energy Compton-scattered $\gamma$ rays which are generated in the materials around the detectors [20]. The relevant centroid difference related only to the full-energy peak (FEP) events is calculated as [14]:

$$
\Delta C_{\mathrm{FEP}}=\Delta C_{\text {Exp. }}+\frac{\Delta C_{\text {Exp. }}-\Delta C_{\mathrm{BG}}}{\Pi}
$$

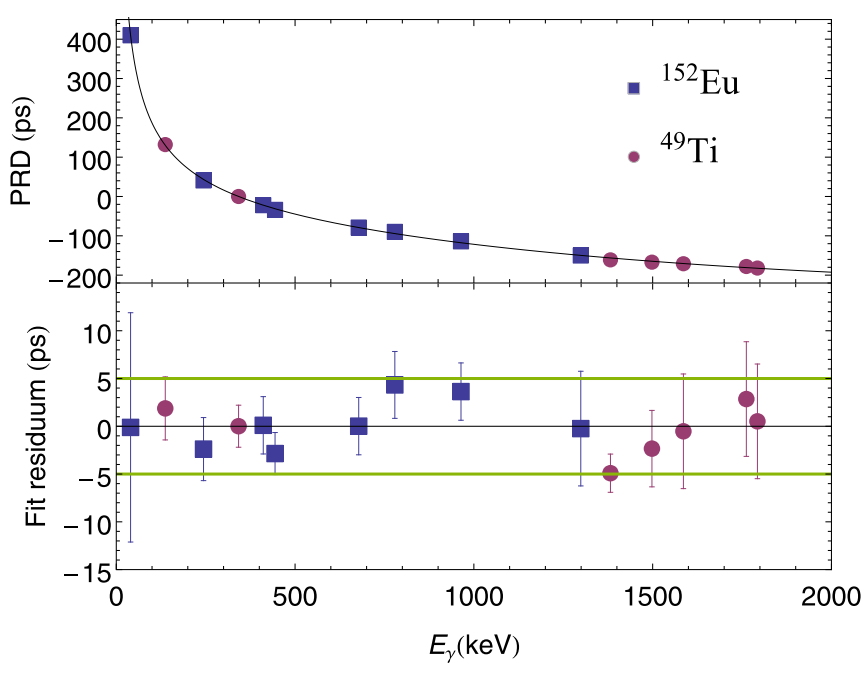

FIG. 3. Top: Energy-dependent mean prompt response difference (PRD) of the FATIMA setup. Bottom: PRD fit residuum from the calibration with the fit function given by Eq. (8). The green lines represent the $3 \sigma$ error-band corresponding to an overall PRD uncertainty of 5 ps.

and the lifetime becomes

$$
\tau=\frac{1}{2}\left(\Delta C_{\text {Exp. }}+\frac{\Delta C_{\text {Exp. }}-\Delta C_{\mathrm{BG}}}{\Pi}-\mathrm{PRD}\right) .
$$

$\Delta C_{\text {Exp. }}$ represents the experimental mean value for the $\mathrm{N}$ detector fast-timing array and includes the FEP and background events, $\Delta C_{\mathrm{BG}}$ is the "time response" of the background, and $\Pi$ is the peak-to-background ratio [18].

Let us consider a nuclear state being fed by a transition $\gamma_{1}$ and decaying via a transition $\gamma_{2} \cdot \gamma_{2}$ will be called reference transition with energy $E_{\gamma_{2}}$. To obtain the lifetime of the state, several analysis steps need to be done:

(i) build the delayed ( $\gamma_{1} \rightarrow$ start and $\gamma_{2} \rightarrow$ stop) and antidelayed $\left(\gamma_{2} \rightarrow\right.$ start and $\gamma_{1} \rightarrow$ stop) time distributions by posing gates in the $\mathrm{LaBr}_{3}(\mathrm{Ce})$ spectra, including the necessary amount of HPGe gates in order to ensure that the right nucleus is selected and determine the centroid difference of the two distributions;

(ii) using the same reference $\gamma_{2}$ line, build delayed and antidelayed time distributions with a second $\mathrm{LaBr}_{3}(\mathrm{Ce})$ gate on energies in the background around $\gamma_{1}$;

(iii) fit the centroid differences of the time distributions between the reference line and the background energies and determine the centroid difference of the background at the energy of $\gamma_{1}$ by interpolation;

(iv) determine the peak-to-background ratio $\Pi$;

(v) determine the prompt response difference of the $\gamma-\gamma$ cascade using Eq. (8) via the transformation PRD = $\operatorname{PRD}\left(E_{\gamma_{1}}\right)-\operatorname{PRD}\left(E_{\gamma_{2}}\right)[19]$.

Thus, all the necessary observables are determined and the lifetime of the nuclear state of interest can be calculated using Eq. (10). The same type of analysis can be done exchanging the roles of $\gamma_{1}$ and $\gamma_{2}$, that is, using $\gamma_{1}$ as a reference transition 

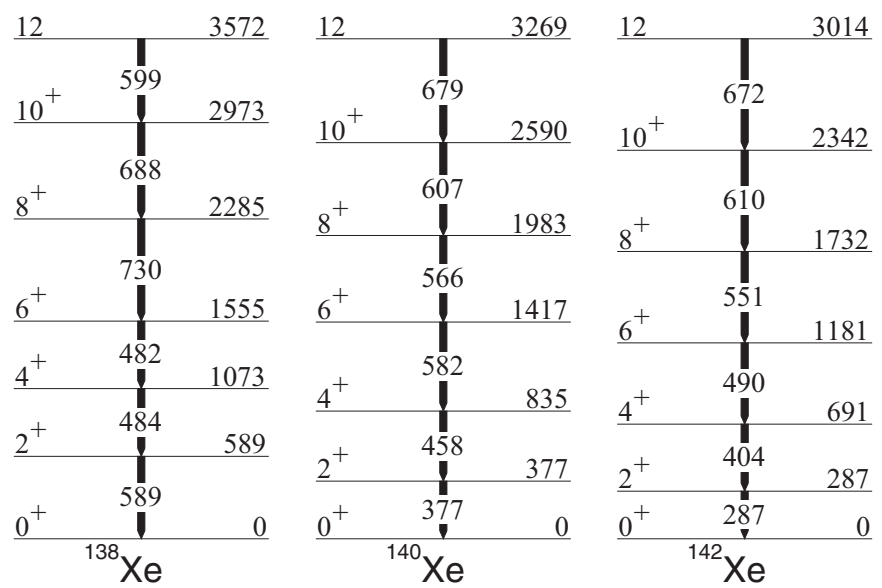

FIG. 4. Partial level schemes of ${ }^{138,140,142}$ Xe. Data from Ref. [22].

and determining the background around $\gamma_{2}$. This analysis gives identical results for the lifetime.

\section{RESULTS}

In this section the results for the lifetimes of excited states in the isotopes ${ }^{138,140,142} \mathrm{Xe}$ will be presented. These isotopes lie close to the high-mass peak of the fission fragment distribution for both ${ }^{235} \mathrm{U}$ and ${ }^{241} \mathrm{Pu}$ targets. The fission yield per 100 fissions for the ${ }^{235} \mathrm{U}$ target is $4.81,3.51$, and 0.434 for ${ }^{138} \mathrm{Xe}$, ${ }^{140} \mathrm{Xe}$, and ${ }^{142} \mathrm{Xe}$, respectively [21]. For the ${ }^{241} \mathrm{Pu}$ target the yields for the same nuclei are 4.06, 3.1, and 0.546, respectively [21].

\section{A. ${ }^{140} \mathrm{Xe}$}

The nucleus ${ }^{140} \mathrm{Xe}$ is the only neutron-rich xenon isotope in which nuclear lifetimes were directly measured previous to our study by Lindroth et al. [2]. In his experiment the excited states in ${ }^{140} \mathrm{Xe}$ were populated following the $\beta$ decay of ${ }^{140} \mathrm{I}$ from a separator and the lifetimes were measured with fast $\mathrm{BaF}_{2}$ scintillators. Therefore, the nucleus was used to test the analysis procedure and the quality of the results that can be obtained with our improved setup keeping in mind that the background conditions were worse.

The partial level scheme of ${ }^{140} \mathrm{Xe}$ is shown in Fig. 4. For the determination of the lifetime of the first $2^{+}$state, time spectra were obtained with a common gate on the transition $582 \mathrm{keV}\left(6^{+} \rightarrow 4^{+}\right)$in the Ge array and a gate on the reference energy $377 \mathrm{keV}\left(2^{+} \rightarrow 0^{+}\right)$in the $\mathrm{LaBr}_{3}(\mathrm{Ce})$ detectors using the data taken with ${ }^{235} \mathrm{U}$ target. Figure 5 shows the resulting double-gated spectra in both detectors arrays. The most prominent peak in these spectra is the $458 \mathrm{keV}$ $\left(4^{+} \rightarrow 2^{+}\right)$transition in ${ }^{140} \mathrm{Xe}$. The delayed and antidelayed time distributions obtained with a second gate on $458 \mathrm{keV}$ in the $\mathrm{LaBr}_{3}(\mathrm{Ce})$ detectors are shown in Fig. 6. The centroid difference of the two distributions is $\Delta C_{\text {Exp. }}=124(7)$ ps.

In Fig. 7 the centroid-difference diagram for the time spectra is shown. The $x$ axis gives the energy of the second $\mathrm{LaBr}_{3}(\mathrm{Ce})$ gate. To determine the timing contribution of the background, the distribution of background centroids was fitted with a second-order polynomial and its value at an

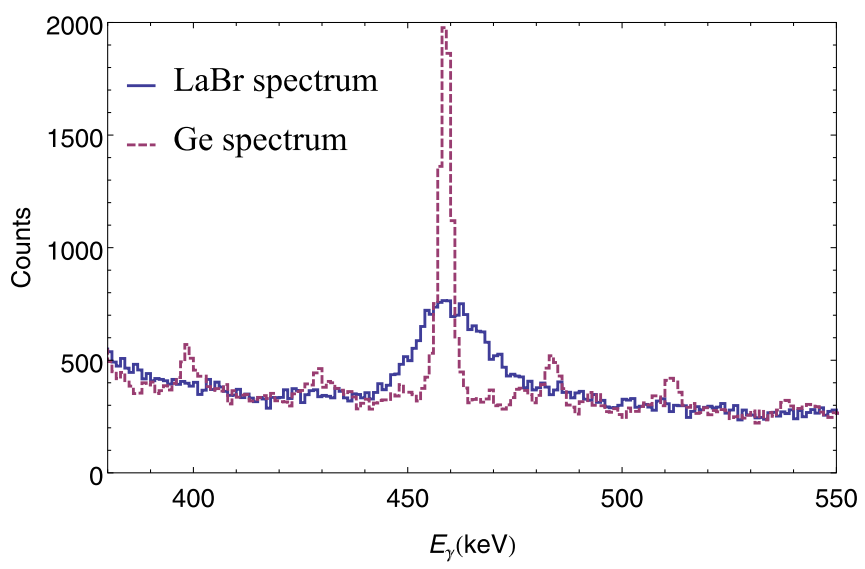

FIG. 5. Energy spectra for ${ }^{140} \mathrm{Xe}$ from the EXILL\&FATIMA array gated on $582 \mathrm{keV}\left(6^{+} \rightarrow 4^{+}\right)$in $\mathrm{Ge}$ and $377 \mathrm{keV}\left(2^{+} \rightarrow 0^{+}\right)$in the $\mathrm{LaBr}_{3}(\mathrm{Ce})$ detectors.

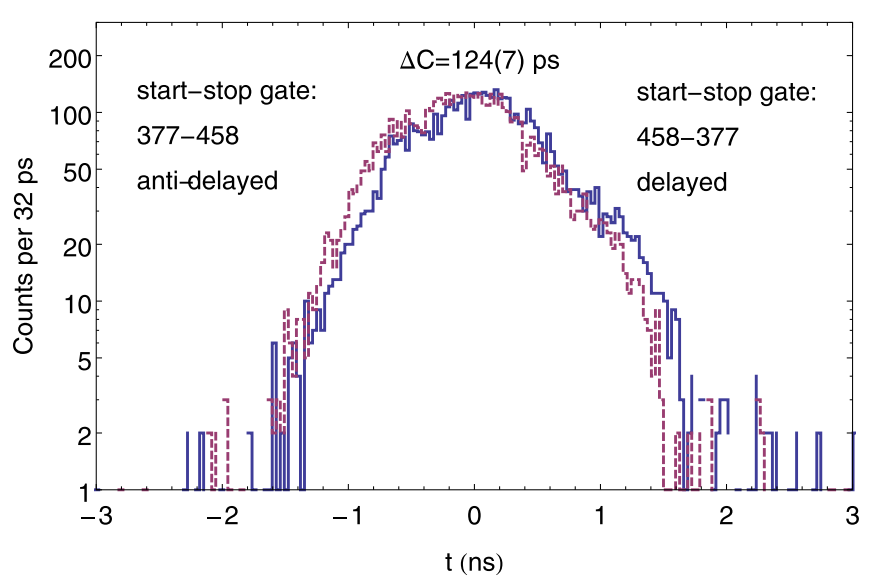

FIG. 6. Delayed (solid blue line) and antidelayed (dashed red line) time distributions obtained with the feeding and decay transitions to the first $2^{+}$state in ${ }^{140} \mathrm{Xe}$ with an additional gate on $582 \mathrm{keV}$ in the EXILL array.

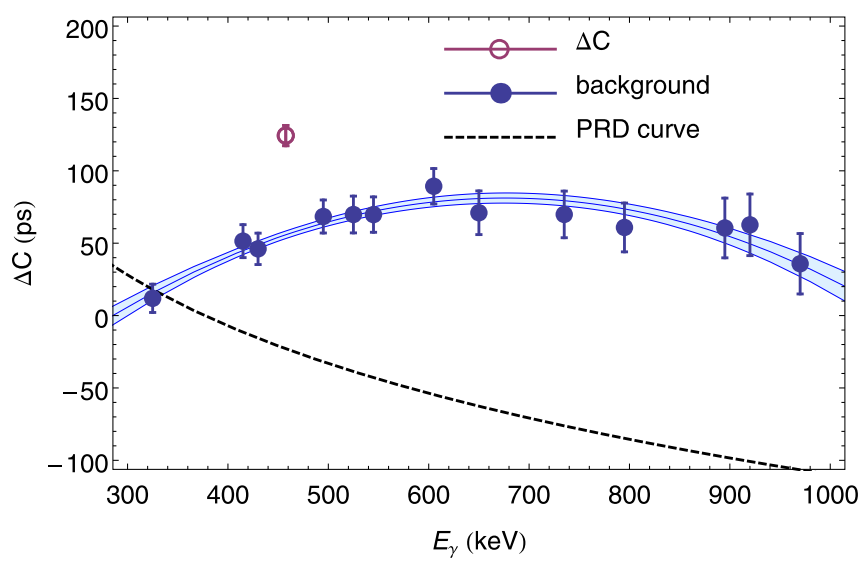

FIG. 7. Centroid-difference diagram for the $2^{+}$state in ${ }^{140} \mathrm{Xe}$. All time spectra were obtained with a common gate at $582 \mathrm{keV}$ in the $\mathrm{Ge}$ detectors and at reference energy $377 \mathrm{keV}$ in the $\mathrm{LaBr}_{3}(\mathrm{Ce})$. The PRD curve is adjusted by a parallel shift to cross the axis at the reference energy. The fit to the background with a second-order polynomial, including $1 \sigma$-error, is shown with a light blue band. 
TABLE I. Mean lifetimes of low-lying excited states in $138,140,142 \mathrm{Xe}$ determined in the EXILL\&FATIMA campaign at ILL using a ${ }^{235} \mathrm{U}$ fission target. For comparison, the previously reported values for the $2^{+}$state in ${ }^{140} \mathrm{Xe}$ are also included.

\begin{tabular}{lccc}
\hline \hline Isotope & $\tau_{2^{+}}(\mathrm{ps})$ & $\tau_{4^{+}}(\mathrm{ps})$ & $\tau_{6^{+}}(\mathrm{ps})$ \\
\hline${ }^{138} \mathrm{Xe}$ & $21(16)$ & & \\
${ }^{140} \mathrm{Xe}$ & $102(7)$ & $17(5)$ & \\
${ }^{140} \mathrm{Xe}^{1}$ & $101.7(32)$ & $22.8(49)$ & $<12.4$ \\
${ }^{140} \mathrm{Xe}^{2}$ & $163(7)$ & & \\
${ }^{142} \mathrm{Xe}$ & $249(23)$ & $54(10)$ & \\
\hline \hline
\end{tabular}

energy of $458 \mathrm{keV}$ was determined as $\Delta C_{\mathrm{BG}}=56(3)$ ps. The prompt response difference is $\mathrm{PRD}=-23(5) \mathrm{ps}$. The peak-to-background ratio was determined from the energy spectrum measured in the $\operatorname{LaBr}_{3}(\mathrm{Ce})$ detectors with the two common gates (see Fig. 5) and it is $\Pi=1.22(2)$. Substituting these values into Eq. (10), one obtains a mean lifetime $\tau_{2^{+}}=102(7) \mathrm{ps}$.

The same analysis was performed for the data taken with the ${ }^{241} \mathrm{Pu}$ target to cross-check the results. The same gates as for the data with the ${ }^{235} \mathrm{U}$ target were used. The obtained centroid differences were $\Delta C_{\text {Exp. }}=96(14)$ ps and $\Delta C_{\mathrm{BG}}=37(9) \mathrm{ps}$. The peak-to-background ratio was determined to be $\Pi=0.75$. In that case, the mean lifetime was $\tau_{2^{+}}=99(18) \mathrm{ps}$, which is consistent with the result obtained with the uranium target. The larger uncertainty reflects the lower statistics from the shorter measurement with the plutonium target.

Further, the lifetime of the $4^{+}$state was also determined using a gate on $377 \mathrm{keV}$ in the Ge detectors and a reference gate on $582 \mathrm{keV}$ in the $\mathrm{LaBr}_{3}(\mathrm{Ce})$ detectors. The result is $\tau_{4^{+}}=17(5)$ ps (Table I). This and all other results presented in Table I were obtained from the measurement with ${ }^{235} \mathrm{U}$ target.

Our results are in agreement with the previous measurement of Lindroth et al. [2]. The larger uncertainties of our measurement are due to the higher background created by the fission products which requires additional gates (reduced statistics) and more corrections (increased systematic uncertainty) than in the cleaner conditions of the experiment studying the $\beta$ decay of ${ }^{140} \mathrm{I}$.

\section{B. ${ }^{142} \mathrm{Xe}$}

${ }^{142} \mathrm{Xe}$ is the most neutron-rich xenon isotope populated in the present experimental campaign with statistics sufficient for determining lifetimes of excited states. For the $2^{+}$state the timing spectra were created with a Ge gate on $490 \mathrm{keV}$ (Fig. 4) and $\mathrm{LaBr}_{3}(\mathrm{Ce})$ gate on $287 \mathrm{keV}$ (reference transition). The centroid-difference diagram is shown in Fig. 8. The mean lifetime was determined to be $\tau_{2^{+}}=249(23) \mathrm{ps}$.

In a similar way, the lifetime of the $4^{+}$state was determined posing gates on $287 \mathrm{keV}$ in the $\mathrm{Ge}$ detectors and $490 \mathrm{keV}$ in the $\operatorname{LaBr}_{3}(\mathrm{Ce})$ detectors (reference $\gamma$ ray). The obtained mean lifetime is $\tau_{4^{+}}=54(10) \mathrm{ps}$.

$$
\text { C. }{ }^{138} \mathrm{Xe}
$$

The situation with ${ }^{138} \mathrm{Xe}$ is more challenging. In the ground-state band there are transitions with similar energies

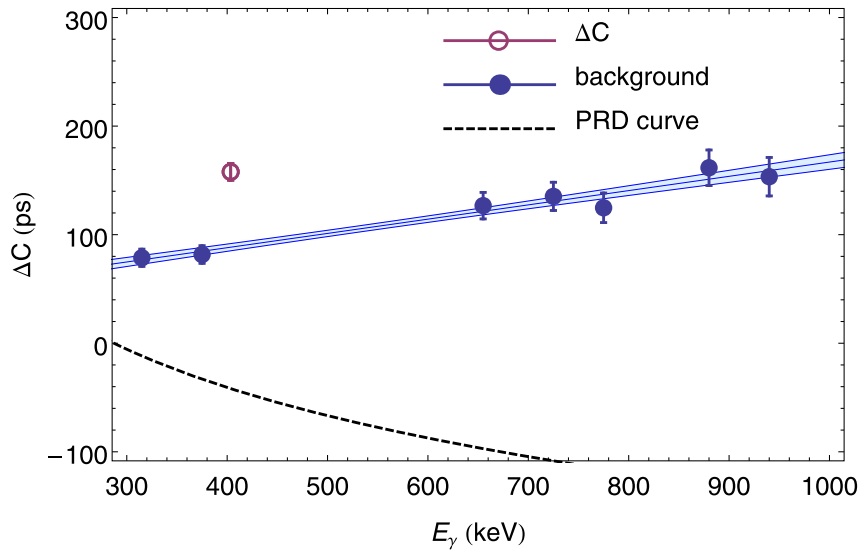

FIG. 8. Centroid-difference diagram for the $2^{+}$state in ${ }^{142} \mathrm{Xe}$. The centroids were obtained with a common gate in the Ge array on 490 $\mathrm{keV}$ and a reference gate in the $\mathrm{LaBr}_{3}(\mathrm{Ce})$ detectors on $287 \mathrm{keV}$. The linear fit to the background including the $1 \sigma$ error band is shown as a light blue band.

(Fig. 4) which make the construction of time spectra difficult or impossible in some cases, i.e., gating on the feeding and decaying transition of the $4^{+}$state which have an energy difference of only $2 \mathrm{keV}$. An attempt was made to determine the lifetime of the $2^{+}$state by posing simultaneously gates on the 482- and 599-keV transitions in the Ge detectors and on 484-keV transition (reference $\gamma$ ray) in the $\mathrm{LaBr}_{3}(\mathrm{Ce})$ detector. The obtained centroid-difference diagram is plotted in Fig. 9. Due to the multiple gates the statistics were very much reduced, which resulted in a higher uncertainty in the extracted lifetime $\tau=21(16)$ ps (see Table I).

The lifetimes of $6^{+}$and higher-lying states could not be determined due to insufficient statistics in both measurements with ${ }^{235} \mathrm{U}$ and ${ }^{241} \mathrm{Pu}$ targets. The octupole properties could not be studied and discussed in this paper as in the experiment in question the negative-parity states in the xenon isotopes were populated with insufficient statistics and no conclusive results could be obtained.

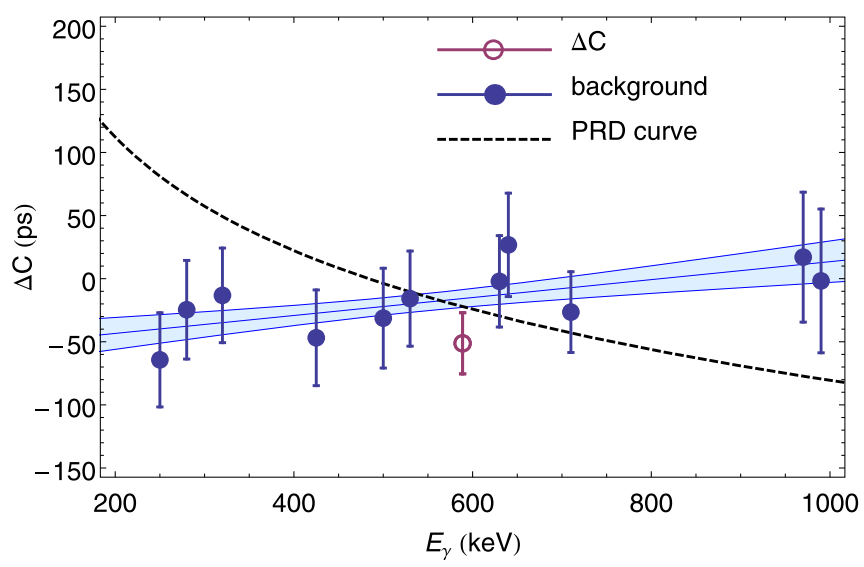

FIG. 9. Centroid-difference diagram for the $2^{+}$state in ${ }^{138} \mathrm{Xe}$. The centroids were obtained with simultaneous gates in the Ge array on $482 \mathrm{keV}$ and $599 \mathrm{keV}$ and a reference gate in the $\mathrm{LaBr}_{3}(\mathrm{Ce})$ detectors on $484 \mathrm{keV}$. The linear fit to the background including the $1 \sigma$ error band is shown as a light blue band. 
TABLE II. Experimental and theoretical $B(E 2 \uparrow)\left(e^{2} b^{2}\right)$ values for the excitation of the first $2^{+}$state in neutron-rich Xe isotopes.

\begin{tabular}{lllcc}
\hline \hline & ${ }^{138} \mathrm{Xe}$ & ${ }^{140} \mathrm{Xe}$ & ${ }^{142} \mathrm{Xe}$ & Ref. \\
\hline Exp. & $0.28(21)$ & $0.53(4)$ & $0.85(8)$ & This work \\
NPA-1 & 0.31 & 0.50 & 0.70 & {$[23]$} \\
NPA-2 & 0.42 & 0.62 & 0.78 & {$[23]$} \\
CD-Bonn & 0.250 & 0.500 & - & {$[24]$} \\
MCSM & 0.257 & 0.451 & - & {$[25]$} \\
LSSM & 0.379 & 0.668 & - & {$[26]$} \\
\hline \hline
\end{tabular}

\section{COMPARISON WITH THEORY}

In Table II the experimental $B(E 2 \uparrow)$ for the excitation of the first $2^{+}$states in ${ }^{138,140,142} \mathrm{Xe}$ are compared to several theoretical calculations. The calculations are all based on the shell model. In Fig. 10 they are compared to the transition strengths calculated from the measured lifetimes and the evolution of $B(E 2 \uparrow)$ values with increasing neutron number for $78 \leqslant N \leqslant 90$ is shown.

Large-scale shell model (LSSM) calculations were performed by Bianco et al. [26]. The shell-model space used for the calculation included the $1 g_{7 / 2}, 2 d_{5 / 2}, 2 d_{3 / 2}, 3 s_{1 / 2}, 1 h_{11 / 2}$ orbitals for protons and $2 f_{7 / 2}, 3 p_{3 / 2}, 1 h_{9 / 2}, 3 p_{1 / 2}, 2 f_{5 / 2}, 1 i_{13 / 2}$ for neutrons. For the two-body potential, a renormalized $G$ matrix derived from the CD-Bonn potential was used. The effective charges used were $e_{\pi}=1.6 e$ and $e_{v}=0.7 e$. The calculation predicts an increasing neutron weight in the lowest collective $2^{+}$state of the isotopes with increasing neutron number. It reproduces reasonably well the experimental values but the increase in collectivity is faster than seen in the experiment.

The shell-model calculations employing a realistic interaction derived from the CD-Bonn nucleon-nucleon potential renormalized within the $V_{\text {low-k }}$ approach [24] (CD-Bonn) agree better with the experimental data for ${ }^{138,140} \mathrm{Xe}$. The outline of the calculations and the used Hamiltonian are

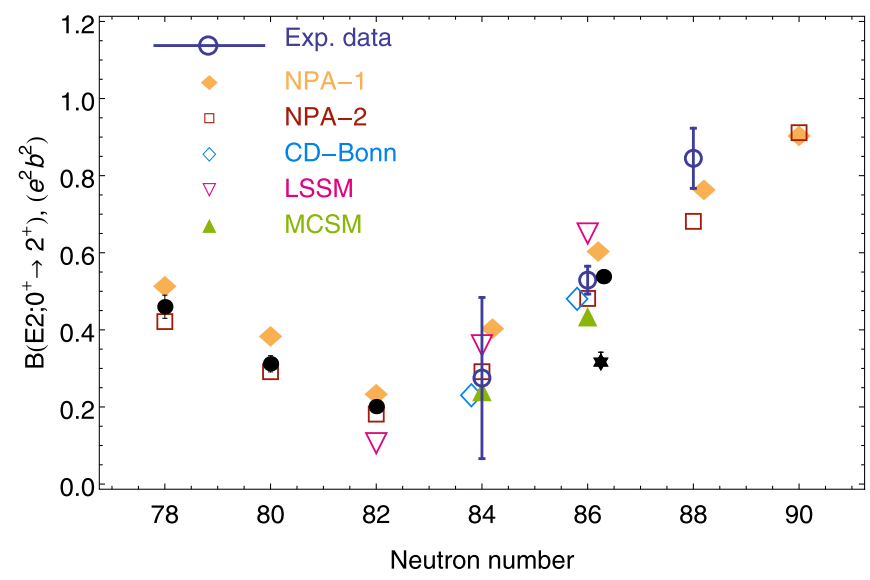

FIG. 10. Experimental and theoretical $B(E 2 \uparrow)$ values in $e^{2} b^{2}$ for even-even Xe isotopes with neutron numbers $78 \leqslant N \leqslant 90$. The experimental values determined in this work are shown with empty circles. The previously reported values are shown with full circles for lighter Xe isotopes [22] and for ${ }^{140} \mathrm{Xe}$ [2]. The black star shows the value for ${ }^{140} \mathrm{Xe}$ reported in Ref. [9]. described in Ref. [27]. The same configuration space as for LSSM calculations was used with a slightly different effective charges: $e_{\pi}=1.55 e$ and $e_{v}=0.7 e$.

The Monte-Carlo shell model (MCSM) [25] describes the evolution of the $B(E 2)$ values with neutron number for ${ }^{140} \mathrm{Xe}$ and ${ }^{142} \mathrm{Xe}$ with acceptable accuracy. The increase in collectivity is interpreted as the "shape" phase transition from spherical vibrator at $N=82$ towards an axially symmetric rotor for $N>82$. The lack of axial asymmetry in this calculation could explain the discrepancy with the experimental value for ${ }^{140} \mathrm{Xe}$ in the light of new evidence of $\gamma$ collectivity found in this nucleus by Urban et al. [28]. A triaxial shape with $(\beta, \gamma)$ parameters $\left(0.15,15.5^{\circ}\right)$ was found to describe the properties of ${ }^{140} \mathrm{Xe}$ better.

A consistent description of the structure of the Xe isotopes for a large range of neutron numbers is given by the nucleonpair approximation (NPA) [23] of the nucleon-pair shell model (NPSM). In the NPSM, nucleon pairs with various angular momenta are used as building blocks of the truncated shell-model space. For low-lying collective excitations in medium and heavy nuclei, a truncation including only $S$ (spin 0 , monopole pairing) and $D$ (spin 2, quadrupole pairing) pairs is sufficient for a good description. The Hamiltonian includes quadrupole-quadrupole interactions between pairs of valence nucleons of the same type and pairs of valence protons and valence neutrons. Thus, using very few parameters, a good description of the experiment is obtained.

The NPA-1 calculation uses the same effective charges for both shells 50-82 and 82-126: $e_{\pi}=1.9389 e$ and $\left|e_{\nu}\right|=$ $1.0795 e$ [23], with $e_{v}$ being positive in the 82-126 shell and negative in the 50-82 shell as the valence neutrons are hole-like in this case. The $B(E 2)$ values calculated in this way are larger than the experimental data near the closed shell and smaller far from the closed shell [23] (see Fig. 10). By using effective charges which change linearly with valence pair number, a better agreement with the experimental data is obtained (NPA-2 calculation, Fig. 10). The effective charges used for NPA-2 calculation were $e_{\pi}=(1+\delta) e$ and $\left|e_{\nu}\right|=\delta e$ with $\delta=0.577+0.0781\left(N_{\pi}+N_{v}\right) . N_{\pi}$ and $N_{v}$ are the numbers of pairs of valence protons and valence neutrons, respectively [23]. The values of the effective charges (parameters for calculating $\delta$ ) for the NPA-1(NPA-2) calculations were obtained by $\chi^{2}$ fitting of the experimental $B(E 2)$ values from Refs. [29,30]. This did not include values for ${ }^{138} \mathrm{Xe}$ and ${ }^{142} \mathrm{Xe}$ where the $B(E 2)$ s were directly determined for the first time in this work. It is interesting to note that even though the fit included the low $B(E 2)$ value determined by Cheifetz et al. [9] for ${ }^{140} \mathrm{Xe}$, which can be discarded following our study, the results are actually consistent with the results from the direct lifetime measurements. The calculations are in agreement with the results presented here. It can be concluded that for the description of the low-lying collective states in xenon the monopole pairing strength, quadrupole pairing strength, and the quadrupole-quadrupole pairing interactions between valence protons, valence neutrons, and valence protons and neutrons are important.

The measured lifetimes have implications on the previous attempt to extract the $g$ factors of the $2^{+}$states in ${ }^{140,142}$ Xe by Goodin et al. [31]. The lifetimes used in Ref. [31] to determine 
the $g$ factors proved to be incorrect. With the new values of $\tau_{2^{+}}$ determined in this work, the following $g$ factors are obtained: $g\left({ }^{140} \mathrm{Xe} ; 2^{+}\right)=0.56(19)$ and $g\left({ }^{142} \mathrm{Xe} ; 2^{+}\right)=0.49(9)$. These values are currently not reproduced by theory calculations (see Refs. [23,31]) which predict much lower values.

\section{CONCLUSION}

Following our study, the discrepant value for the $B(E 2 \uparrow)$ for the $2^{+}$state in ${ }^{140} \mathrm{Xe}$ determined by Cheifetz et al. [9] can now be discarded. The new measurements show that the quadrupole collectivity ( $B(E 2)$ values) above the closed shell $N=82$ in the xenon isotopic chain increase smoothly with increasing neutron number. This evolution is similar to the one showed by the measured masses [8], where, unlike the higher $Z$ even-even nuclei, there is no unusual behavior in the xenon chain. With the newly measured lifetimes the values determined for the $g$ factors of the $2^{+}$states in ${ }^{140} \mathrm{Xe}$ and ${ }^{142} \mathrm{Xe}$ could also be revised.

\section{ACKNOWLEDGMENTS}

This work is supported by NuPNET and the German ministry of education and research (BMBF) under Grants No. 05P12RDCIA, No. 05P12RDNUP, and No. 05P12PKNUF; by ILL; and by HIC for FAIR. Also supported by the UK Science and Technology Facilities Council and the UK National Measurement Office. The authors appreciate the support of several services at ILL and LPSC.
[1] Th. Kröll et al., AIP Conf. Proc. 1012, 84 (2008).

[2] A. Lindroth, B. Fogelberg, H. Mach, M. Sanchez-Vega, and J. Bielčík, Phys. Rev. Lett. 82, 4783 (1999).

[3] W. Urban et al., Eur. Phys. J. A 16, 303 (2003).

[4] R. Casten, Nuclear Structure from a Simple Perspective (Oxford University Press, Oxford, 1998).

[5] W. Nazarewicz and S. L. Tabor, Phys. Rev. C 45, 2226 (1992).

[6] P. A. Butler and W. Nazarewicz, Nucl. Phys. A533, 249 (1991).

[7] B. Bucher, S. Zhu, C. Y. Wu, R. V. F. Janssens, D. Cline, A. B. Hayes, M. Albers, A. D. Ayangeakaa, P. A. Butler, C. M. Campbell, M. P. Carpenter, C. J. Chiara, J. A. Clark, H. L. Crawford, M. Cromaz, H. M. David, C. Dickerson, E. T. Gregor, J. Harker, C. R. Hoffman, B. P. Kay, F. G. Kondev, A. Korichi, T. Lauritsen, A. O. Macchiavelli, R. C. Pardo, A. Richard, M. A. Riley, G. Savard, M. Scheck, D. Seweryniak, M. K. Smith, R. Vondrasek, and A. Wiens, Phys. Rev. Lett. 116, 112503 (2016).

[8] D. Neidherr, R. B. Cakirli, G. Audi, D. Beck, K. Blaum, C. Bohm, M. Breitenfeldt, R. F. Casten, S. George, F. Herfurth, A. Herlert, A. Kellerbauer, M. Kowalska, D. Lunney, E. Minaya-Ramirez, S. Naimi, M. Rosenbusch, S. Schwarz, and L. Schweikhard, Phys. Rev. C 80, 044323 (2009).

[9] E. Cheifetz, H. A. Selic, A. Wolf, R. Chechik, and J. B. Wilhelmy, in Proc. Conf. Nucl. Spectr. Fission Products, 1980 (1980) p. 193, http://www.nndc.bnl.gov/nsr/nsrlink. jsp?1980ChZM.

[10] I. Ahmad and W. R. Phillips, Rep. Prog. Phys. 58, 1415 (1995).

[11] W. Andrejtscheff, M. Senba, N. Tsoupas, and Z. Z. Ding, Nucl. Instr. Methods 204, 123 (1982).

[12] A. Dewald, O. Möller, and P. Petkov, Prog. Part. Nucl. Phys. 67, 786 (2012).

[13] W. Urban et al., J. Instrum. 8, P03014 (2013).

[14] J.-M. Régis, G. S. Simpson et al., Nucl. Instr. Methods A 763, 210 (2014).
[15] N. Saed-Samii, Diploma thesis, Universität zu Köln, Institut für Kernphysik, 2013.

[16] J.-M. Régis et al., Nucl. Instr. Methods A 726, 191 (2013).

[17] Z. Bay, Phys. Rev. 77, 419 (1950).

[18] J.-M. Régis et al., Nucl. Instr. Methods A 622, 83 (2010).

[19] J.-M. Régis, N. Saed-Samii et al., Nucl. Instr. Methods A 823, 72 (2016).

[20] J.-M. Régis et al., Nucl. Instr. Methods A 811, 42 (2016).

[21] T. R. England and B. F. Rider, Los Alamos National Laboratory report LA-UR-94-3106, ENDF (1994).

[22] National nuclear data center, Accessed: August 23, 2016.

[23] L. Y. Jia, H. Zhang, and Y. M. Zhao, Phys. Rev. C 75, 034307 (2007).

[24] A. Gargano (private communication).

[25] N. Shimizu, T. Otsuka, T. Mizusaki, and M. Honma, J. Phys. Conf. Ser. 49, 178 (2006).

[26] D. Bianco, N. Lo Iudice, F. Andreozzi, A. Porrino, and F. Knapp, Phys. Rev. C 88, 024303 (2013).

[27] B. F. Bayman, A. Covello, A. Gargano, P. Guazzoni, and L. Zetta, Phys. Rev. C 90, 044322 (2014).

[28] W. Urban, K. Sieja, T. Rzaca-Urban, M. Czerwinski, H. Naidja, F. Nowacki, A. G. Smith, and I. Ahmad, Phys. Rev. C 93, 034326 (2016).

[29] S. Raman, C. W. Nestor Jr., and P. Tikkanen, At. Data Nucl. Data Tables 78, 1 (2001).

[30] D. C. Radford, C. Baktash, J. R. Beene, B. Fuentes, A. Galindo-Uribarri, C. J. Gross, P. A. Hausladen, T. A. Lewis, P. E. Mueller, E. Padilla, D. Shapira, D. W. Stracener, C. H. Yu, C. J. Barton, M. A. Caprio, L. Coraggio, A. Covello, A. Gargano, D. J. Hartley, and N. V. Zamfir, Phys. Rev. Lett. 88, 222501 (2002).

[31] C. Goodin, J. R. Stone, N. J. Stone, A. V. Ramayya, A. V. Daniel, J. H. Hamilton, K. Li, J. K. Hwang, G. M. Ter-Akopian, and J. O. Rasmussen, Phys. Rev. C 79, 034316 (2009). 